\title{
Análisis del impacto económico del PIB en Centroamérica, utilizando sistemas de información geográfica, en un período del 2010 al 2014
}

Jessica Gabriela Villatoro Escobar

Yeny Maribel Castellanos Zelaya

\section{Resumen}

El objetivo fue analizar el impacto económico del Producto Interno Bruto (PIB) en Centroamérica utilizando Sistemas de Información Geográfica (SIG). Metodológicamente se realizó un enfoque cuantitativo consistiendo en la recolección de datos numéricos e identificación de las variables que influyen sobre el PIB. Utilizando como muestra, la tasa de crecimiento en un período del 2010 al 2014 en la Región Centroamericana, y el cálculo de los parámetros de medición de esta tasa de crecimiento. Además, los Sistemas de Información Geográfica en la técnica de agrupación de datos para expresar el PIB por año, en donde los datos estadísticos,así como el análisis estadístico del conjunto de variablesse relacionaron con la información georreferenciada de la cartografía base existente de la región. Los resultados obtenidos muestran los niveles de producción de cada país, el gasto por consumo en promedio que se tienen de cada región y gobiernos. Se concluye que los niveles de producción, las exportaciones e importaciones y el consumo, desempeñan un papel fundamental en la economía de un país. El consumo y la inversión pueden fluctuar de un año a otro, en el caso de inversión las variaciones pueden ser bastantes grandes. Lo anterior expresa que no está garantizado que el flujo total de gasto monetario, crecerá de una manera uniforme de un año a otro. En general, la región Centroamericana muestra disminución en los niveles de producción y de consumo de la población, así mismo un incremento en las tasas de desempleo de los países que la conforman.

Palabras clave: Tasa de crecimiento, niveles de producción, gasto por consumo, exportaciones, importaciones. 


\section{Abstract}

The objective was to analyze the economic impact of ProductolnternoBruto (PIB) in Central America using Geographic Information Systems (GIS). Methodologically a quantitative approach consisting of numerical data collection and identification of variables that influence the PIB was performed. Using as a sample, the growth rate in a period from 2010 to 2014 in the Central Region, and the calculation of the measurement parameters of this growth rate. Besides the GIS in data clustering technique to express PIB per year, where the statistical data and the statistical analysis of all variables related to the geo-referenced information from the existing base mapping of the region. The results showed production levels of each country, the average consumption expenditure we have of each region and governments. It is concluded that the levels of production, exports and imports and consumption play a key role in the economy of a country. Consumption and investment can fluctuate from year to year, investment in the case of variations can be quite large. This states that it is not guaranteed that the total flow of money spending, grow a uniform manner from year to year. In general, the Central region shows decreased levels of production and consumption of the population, also an increase in unemployment rates in the countries that comprise it.

Keywords: growth rate, levels of production, consumption expenditure, exports, imports.

Jessica Gabriela Villatoro Escobar, (jessicavillatoro_escobar@yahoo.com). Yeny Maribel Castellanos Zelaya, (yeny.castell@gmail.com) .Departamento de Ciencia y Tecnologías de la Información Geográfica. Facultad de Ciencias Espaciales, Universidad Nacional Autónoma de Honduras. 


\section{INTRODUCCIÓN}

Todos los países experimentan altos y bajos en su crecimiento económico, es decir que la tasa de crecimiento varía tanto en el tiempo como entre los países, sus fluctuaciones tienden a correlacionarse entre sí, pero algunos países experimentan mayor volatilidad en sus tendencias que otros y las diferencias permanecen durante algunos años.

El producto interno bruto (PIB) está conformado por los bienes y servicios producidos en un país por lo que es necesario que se tome en cuenta como medida del bienestar material de una sociedad y es objeto de estudio de la macroeconomía (Doménech, 2004).

Fue de vital importancia llevar a cabo el análisis del impacto del PIB en Centro América (CA) utilizando Sistemas de Información Geográfica (SIG), porque permite a los entes nacionales e internacionales conocer el grado de crecimiento del desarrollo de su país, sopesar que sectores están en crecimiento y que sectores han disminuidos, y buscar las causas, bien sea, por fenómenos naturales o por consecuencias de restricciones de políticas de gobierno. Entre varios asuntos, el estudio nos permite cuantificar el costo de vida, lo cual es de mucha importancia ya que, si no existiera la cuantificación de costo de vida por medio del PIB, no habría anualmente un aumento en las prestaciones de seguridad social, ni en el salario mínimo legal vigente.

Por otro lado el hecho de relacionar el PIB geográficamente causa relevancia ya que los Sistemas de Información Geográfica (SIG) son en la actualidad una valiosa herramienta de análisis geoespacial y de cálculo matemático, que ha empezado a ser usada en varias áreas temáticas, por lo que ocupan un lugar preponderante al ingresar en el ámbito de la planificación y toma de decisiones vinculando tecnologías existentes y ampliando sus posibilidades a través de la incorporación de conceptos y métodos geográficos aplicados a la economía enfocada en el consumo, la producción, las exportaciones, importaciones, el gasto, la inversión; todo esto representado mediante un análisis espacial.(Klein L. , S/F)

\section{METODOLOGÍA}

Para una adecuada organización, análisis e interpretación del impacto económico del PIBsobre el territorio centroamericano, se desarrollaron cálculos estadísticos sobre los datos utilizando programas especializados en bases de datos y en SIG. 
En tal sentido, a continuación, se muestra la estructura lógica de la metodología, con la cual se alcanzaron los objetivos propuestos anteriormente.

\section{Enfoque y Muestra}

El enfoque del estudio fue cuantitativo, el cual consistió en la recolección de datos y análisis estadístico de un conjunto de variables independientes (consumo, gasto, inversión, exportaciones e importaciones) y que influyen sobre el PIB (variable dependiente). Fueron recolectados datos del año 2010 al 2014, a través de los cuales se determinaron tasas de crecimiento por cada variable (ver Tabla 1), por lo que se determinógeográficamente la tendencia del PIB en el tiempo.

La muestra hizo referencia en la unidad de análisis del territorio centroamericano y su tasa de crecimiento por país, en un periodo de (2010-2014).

Tabla 1. Descripción de Variables

\begin{tabular}{|l|l|}
\hline \multicolumn{1}{|c|}{ Variables } & \multicolumn{1}{c|}{ Descripción } \\
\hline PIB & $\begin{array}{l}\text { Es una magnitud macroeconómica que expresa el valor monetario de la producción de } \\
\text { bienes y servicios de demanda final de un pais durante un periodo determinado de tiempo. } \\
\text { (Parkin, Esquivel, \& Muñoz, 2008) }\end{array}$ \\
\hline Consumo & $\begin{array}{l}\text { Es la parte de la renta que se destina a la adquisición de bienes y servicios para la } \\
\text { satisfacción de necesidades. La parte de la renta que no se consume constituye el ahorro } \\
\text { del periodo. Cuando el consumo rebasa la renta de un determinado periodo de tiempo, el } \\
\text { correspondiente agente económico tendrá que endeudarse; en ese caso, el ahorro del } \\
\text { periodo es negativo. (Parkin, Esquivel, \& Muñoz, 2008) }\end{array}$ \\
\hline Inversión & $\begin{array}{l}\text { Formación o incremento neto de capital. La inversión de un determinado periodo de tiempo } \\
\text { viene dada por la diferencia entre el capital existente al final y al comienzo de dicho } \\
\text { periodo; diferencia que puede ser positiva o negativa, según que haya habido inversión 0 } \\
\text { desinversión, respectivamente (Parkin, Esquivel, \& Muñoz, 2008) }\end{array}$ \\
\hline Gasto público & $\begin{array}{l}\text { Es la suma de los gastos realizados por las instituciones, entidades y organismos } \\
\text { integrantes del sector público de una economía nacional. (Parkin, Esquivel, \& Muñoz, } \\
\text { 2008) }\end{array}$ \\
\hline Exportaciones & $\begin{array}{l}\text { Se refiere aquella actividad comercial a través de la cual un producto o un servicio se } \\
\text { venden en el exterior, es decir, a otro pais o paises. (Parkin, Esquivel, \& Muñoz, 2008) }\end{array}$ \\
\hline Importaciones & $\begin{array}{l}\text { Es la acción comercial que implica y desemboca en la introducción de productos foráneos } \\
\text { en un determinado pais con la misión de comercializarlos. (Parkin, Esquivel, \& Muñoz, } \\
\text { 2008) }\end{array}$ \\
\hline
\end{tabular}




\section{Descripción de instrumentos y Técnicas}

\section{- Gestión de la información}

Consistió en los métodos y procedimientos para el tratamiento de la información que se recolecto de bases de datos y memorias obtenidas de cada entidad responsable de emitir los datos económicos por país de Centroamérica, por lo general, del banco central.

De la información recolectada se revisaron, depuraron y analizaron las bases de datos, utilizando como técnica la agrupación de datos, que expresan el Producto Interno Bruto por año establecido y para cada una de las variables independientes y dependiente.Proceso necesario para estandarizar los términos económicos manejados por las diferentes entidades. Posteriormente en programas especializados en SIG, se calcularon tasas de crecimiento también llamada tasa de cambio, mediante la siguiente fórmula:

\section{Variación porcentual $=($ valor presente-valor pasado $) /$ valor pasado}

De la fórmula planteada resultaron valores de variables independientes (consumo, gasto, inversión, importaciones, exportaciones) y el PIB que es una variable dependiente obtenida del conjunto de variables independientes.

\section{- $\quad$ Representación}

Una vez realizados los cálculos, se procedió a categorizar mediante escalas de medición ordinal, los resultados positivos y negativos por país, organizándolos en diferentes tablas de atributos, una por variable, creando así diferentes capas de información. Además de tablas de Excel, con el fin de generar gráficos de tendencia alusivos al PIB y sus componentes.

Posteriormente se realizó el cruce de capas de información, generando un análisis espacial con SIG, mediante la aplicación de una técnica de representación por mapas de puntos y Coropletas, obteniendo como producto final diferentes mapas temáticos comparativos que muestran representación geográfica del PIB y sus componentes para su posterior análisis y discusión. 


\section{- $\quad \underline{\text { Análisis }}$}

El impacto económico se analizó de forma global y tendencial, esto desde el punto de vista del consumo, el gasto, inversión, exportaciones e importaciones, así mismo del PIB, englobando todas las variables independientes.

La cartografía obtenida de programas especializados en Sistemas de Información Geográfica, permitió hacer un análisis comparativo de los diferentes valores relativos resultantes.

El análisis se realizó siguiendo los indicadores que a continuación se enumeran:

- valores positivos, aumento de colores, tamaño de puntos grandes (indican crecimiento, es decir, impacto positivo para la economía de un país)

- valores negativos, disminución de colores, tamaño de puntos pequeños (indican decrecimiento, es decir, impacto negativo para la economía de un país)

\section{RESULTADOS Y DISCUSIÓN}

\section{Tasa de crecimiento del PIB en CA y sus componentes}

A través del programa Excel se generaron gráficos que permitieran analizar la tendencia del periodo 2010-2014, en tal sentido se obtuvieron los siguientes resultados:

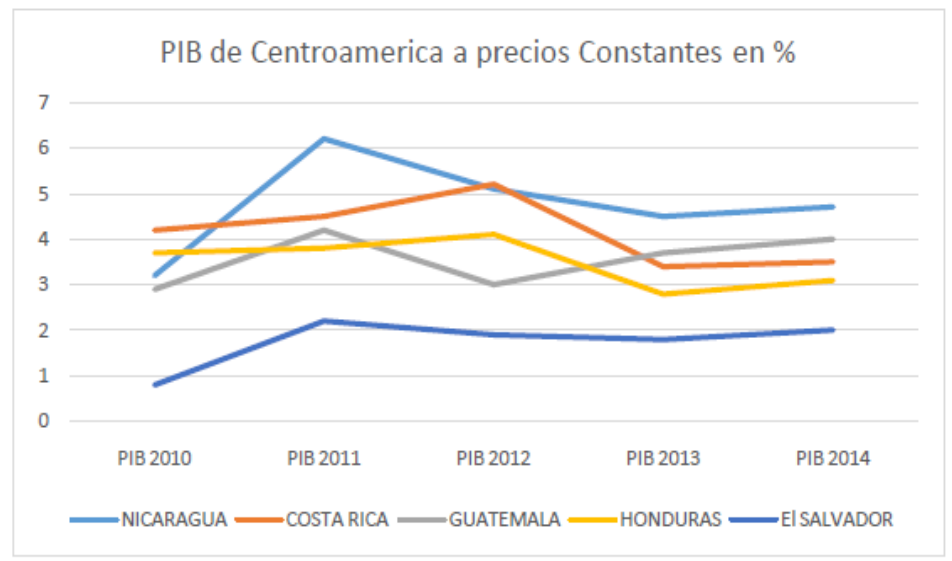

Figura 1. Fuente propia. Datos obtenidos de FMI (Fondo Monetario Internacional) 
El mayor crecimiento económico durante el periodo 2010-2014 (Figura 1) lo tiene Nicaragua con $1.5 \%$, en cambio Costa Rica y Honduras presentan una des aceración de 0.7 y 0.6 en el orden respectivo, en términos generales se observa que Centroamérica su crecimiento económico es muy lento, provocando que no existan suficientes fuentes de empleo para la población, generando un deterioro en la calidad de vida de los habitantes de esta región y un estancamiento en el desarrollo humano.

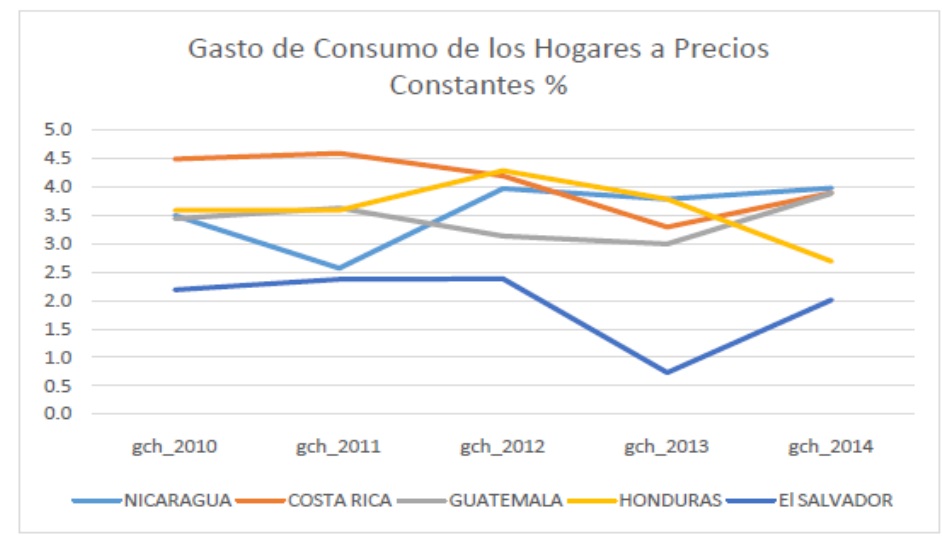

Figura 2. Fuente propia. Datos obtenidos de FMI (Fondo Monetario Internacional)

Durante el periodo analizado (Figura 2), el mayor gasto en bienes y servicios lo tiene Nicaragua con un incremento de $0.5 \%$, en lo que respecta Costa Rica, Honduras y el salvador las familias disminuyeron su consumo en $0.6 \% ; 0.9 \% ; 0.2 \%$ en su orden respectivo, esto se debe al incremento en el costo de vida en el tiempo transcurrido.

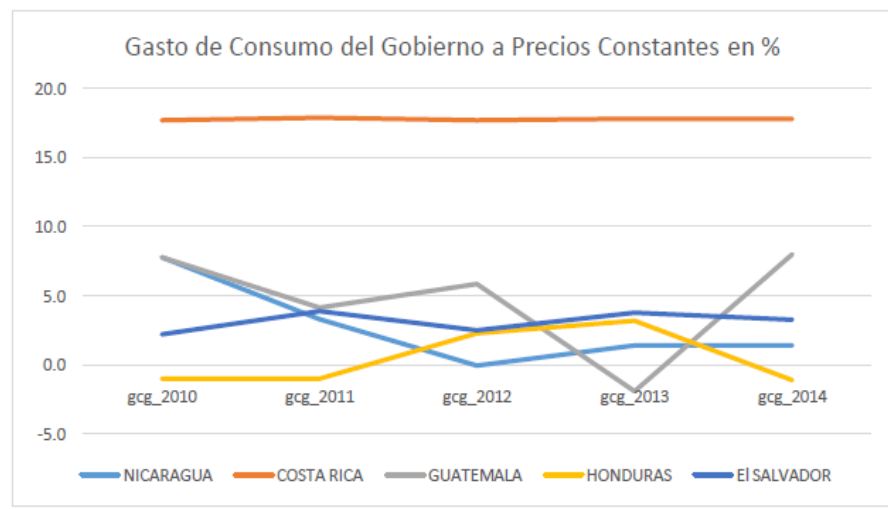

Figura 3. Fuente propia. Datos obtenidos de FMI (Fondo Monetario Internacional) 
Durante el período comprendido 2010-2014 (Figura 3) el gobierno de Nicaragua y Honduras ha disminuido su gasto en $6.4 \%$ y $0.1 \%$, el Gobierno de Costa Rica ha mantenido su gasto Constante en $0.1 \%$, el gobierno de Guatemala y el Salvador incrementaron su consumo en $0.2 \%$ y $1 \%$, este comportamiento se debe a la ejecución de la política fiscal impuesta en cada país.

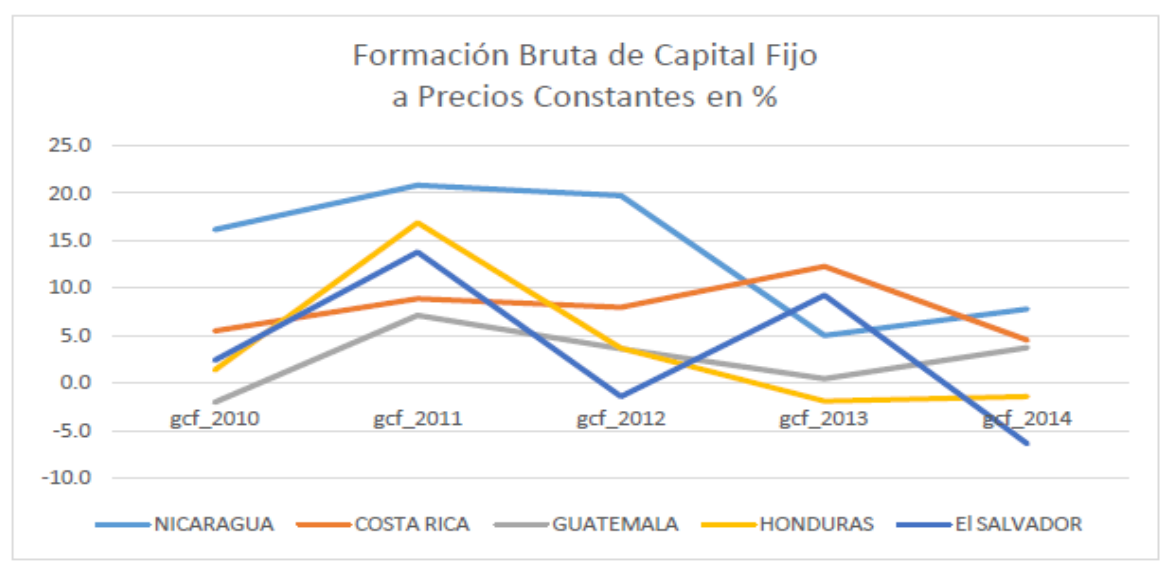

Figura 4. Fuente propia. Datos obtenidos de FMI (Fondo Monetario Internacional)

(Figura 4) El salvador, Nicaragua, Honduras, Costa Rica disminuyeron su Inversión en $8.8 \% ; 8.3 \% ; 2.8 \% ; 1 \%$, en cambio en Guatemala creció en $5.8 \%$, esto se debió al incremento durante el periodo de los precios en la materia prima, de la maquinaria y equipo, entre otros.

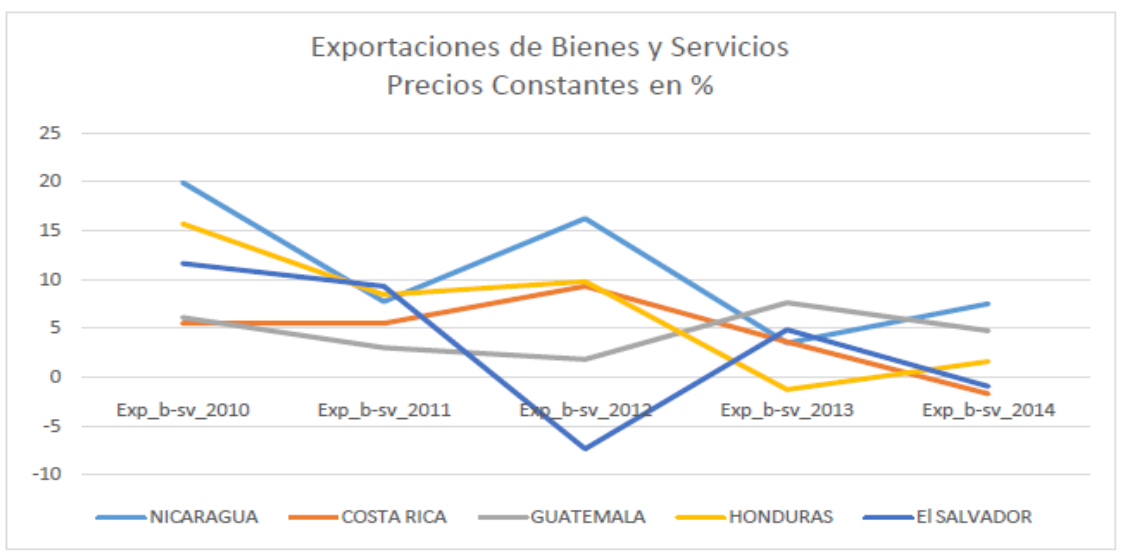

Figura 5. Fuente propia. Datos obtenidos de FMI (Fondo Monetario Internacional) 
Durante el período 2010-2014 (Figura 5) el país que más ha disminuido sus exportaciones ha sido Honduras, lo cual se debe a una disminución del consumo mundial de un $14.1 \%$, sobre todo de los principales socios comerciales. Igual comportamiento presenta el resto de Centroamérica.

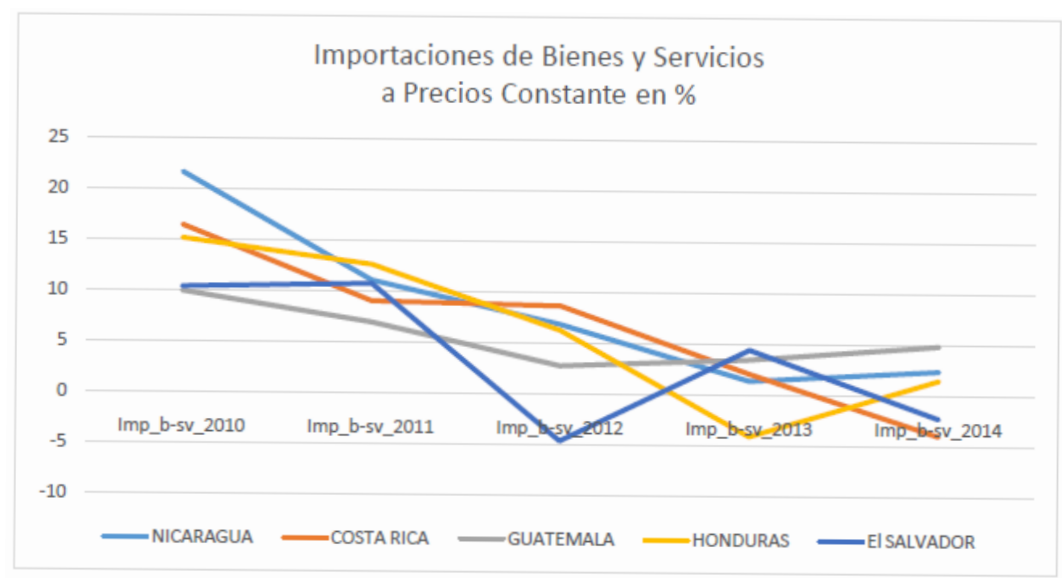

Figura 6. Fuente propia. Datos obtenidos de FMI (Fondo Monetario Internacional)

En términos generales durante el periodo 2010-2014 (Figura 6) el consumo por bienes y servicios importados ha disminuido en toda la región centroamericana, debiéndose al incremento del costo de vida, de la tasa de desempleo y a las secuelas que ha dejado la recesión mundial del 2009 -2015.

\section{Representación geográfica del PIB de CA y sus componentes}

A través del programa ArcGis 10.2 se incorporó a la base de datos espacial la información recopilada, organizada y clasificada, generando finalmente un análisis y representación geográfica del impacto económico del PIB y los componentes que lo conforman y que varían de un año a otro según sus fluctuaciones y sus comportamientos, es así que se obtuvieron como resultados los siguientes mapas. 


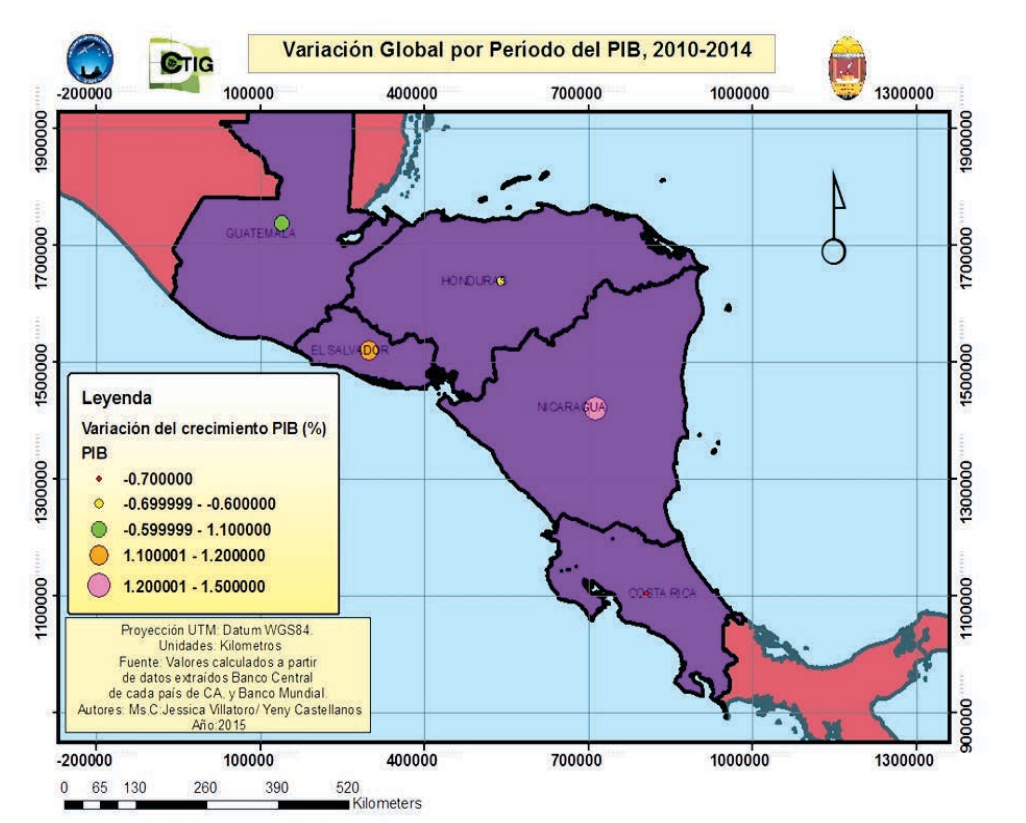

Figura 7: Muestra valores bajos de - 0.7 para Costa Rica mostrando desaceleración y Valores de 1.2 a 1.5 para Nicaragua. Esto indica que el en el período 2010-2014 el mayor crecimiento económico lo tiene Nicaragua.

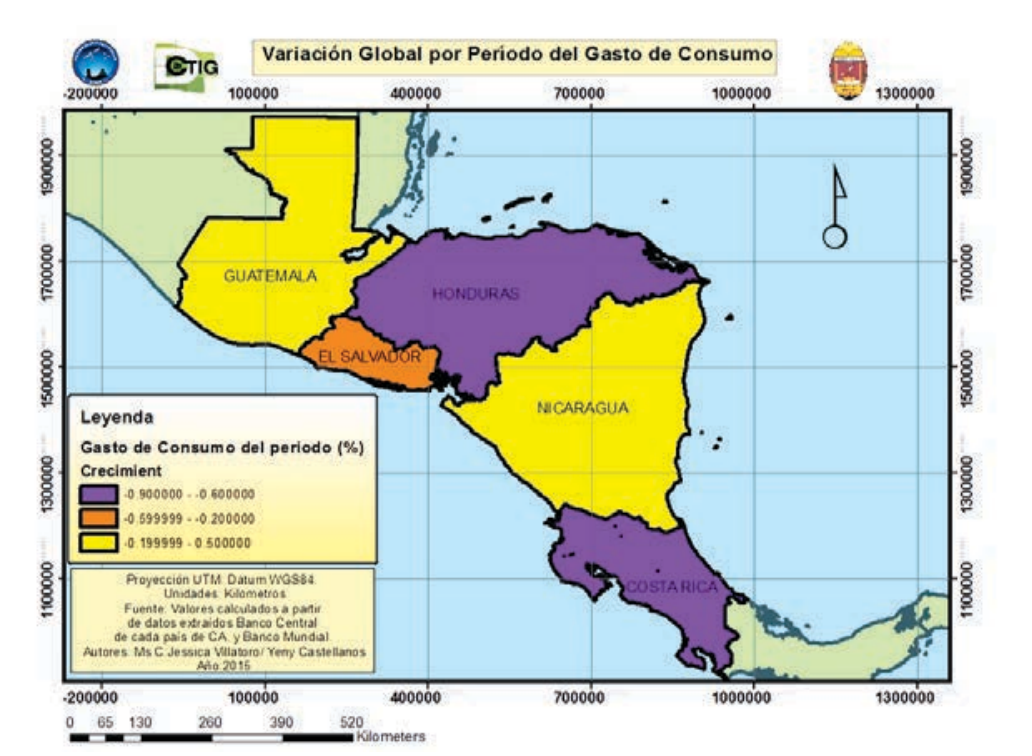

Figura 8: Los valores $(-0.19,0.5)$ indican mayores gastos de bienes y servicios en Nicaragua, en cambio en Honduras y Costa Rica las familias disminuyeron su consumo $(-0.9,-0.6)$ 


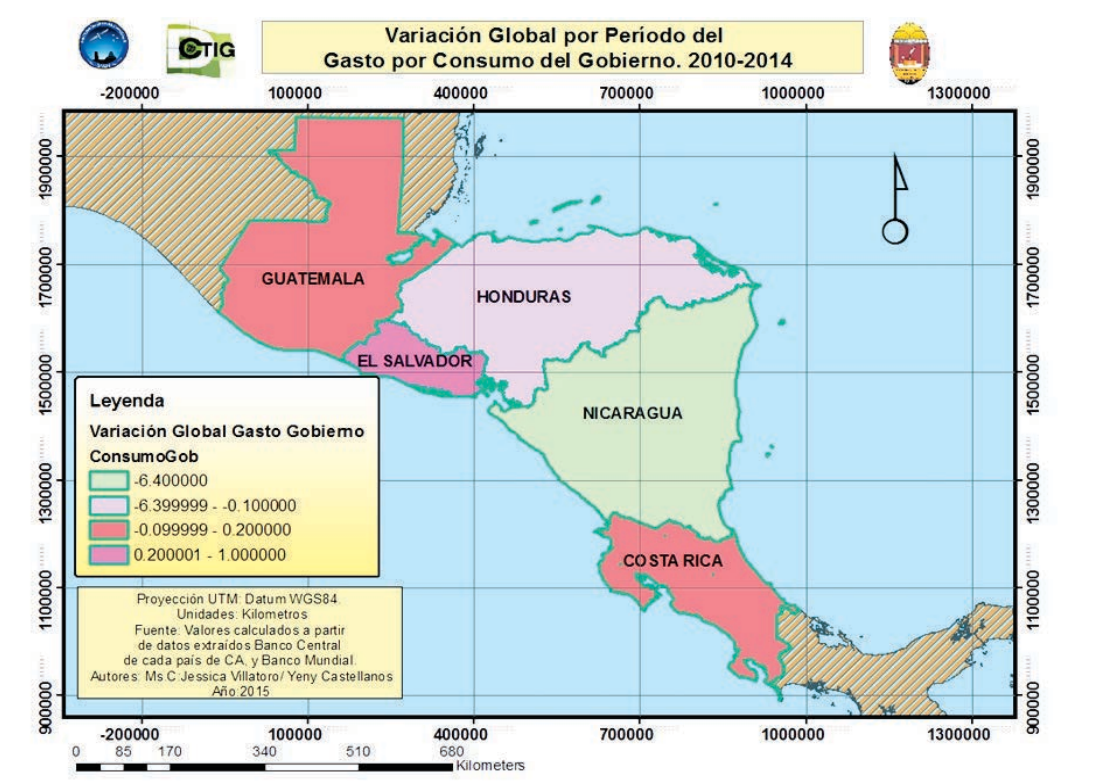

Figura 9 : En cuanto al gasto por consumo del Gobierno (imagen 3). En el período 2010-2014 Nicaragua con valores de -6.4 indica que ha disminuido su gasto por parte del gobierno, en cambio en El Salvador $(0.2,1)$ el gobierno incremento su gasto por consumo.

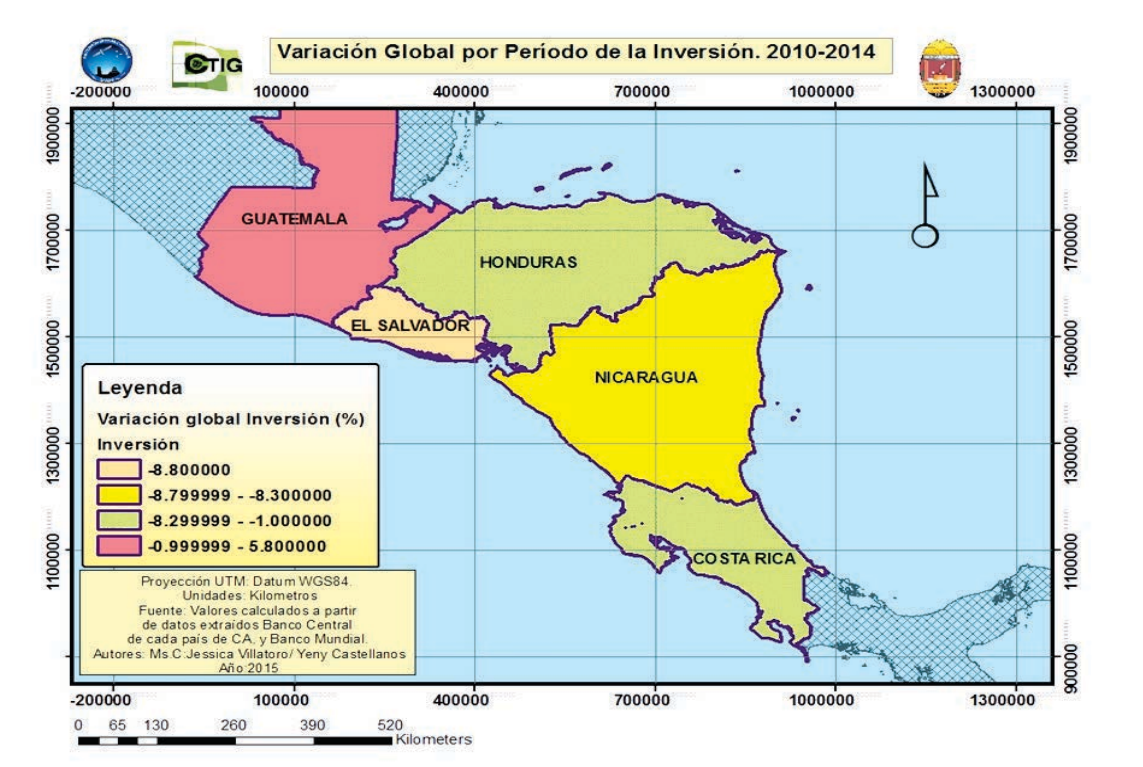

Figura 10: muestra que, desde el punto de vista de la inversión, todos los países disminuyeron su inversión, a excepción de Guatemala que incremento su inversión (-0.9 \%, 5.8\%) 


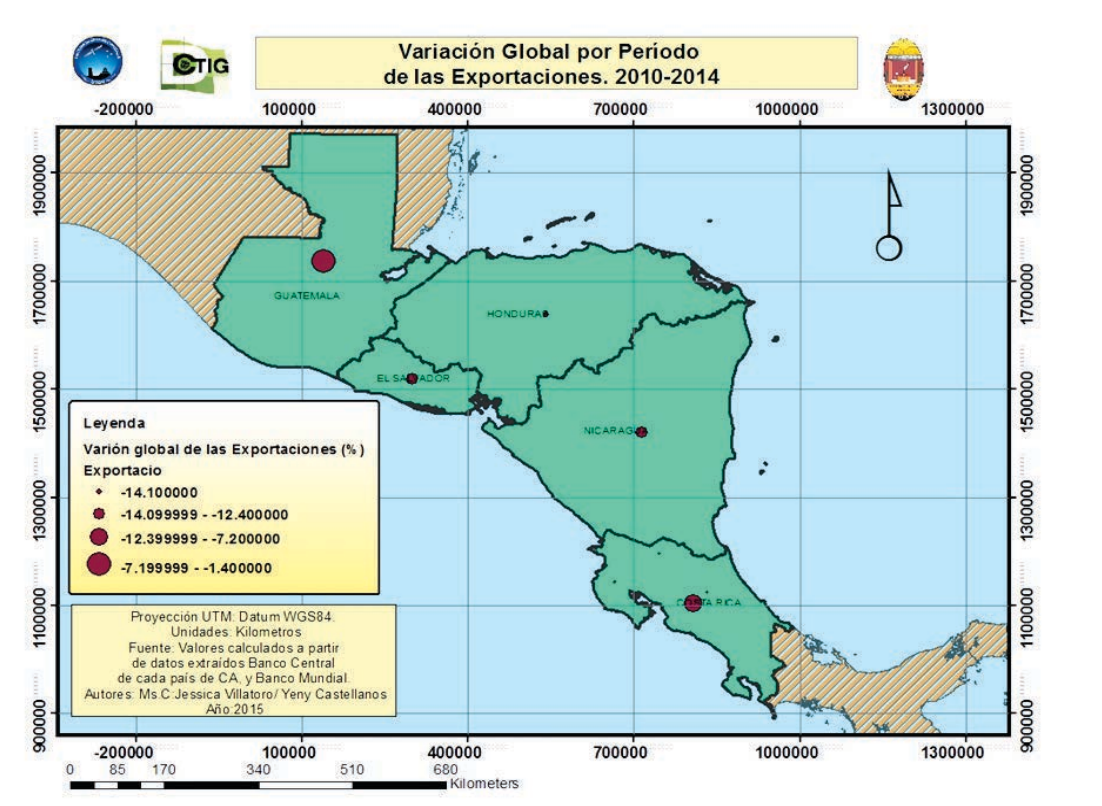

Figura 11: El punto más pequeño lo refleja Honduras (-14.1\%) lo que refleja que durante el período 2010-2014 el país de Honduras disminuyo sus exportaciones en comparación al resto de países de la región Centroamericana.

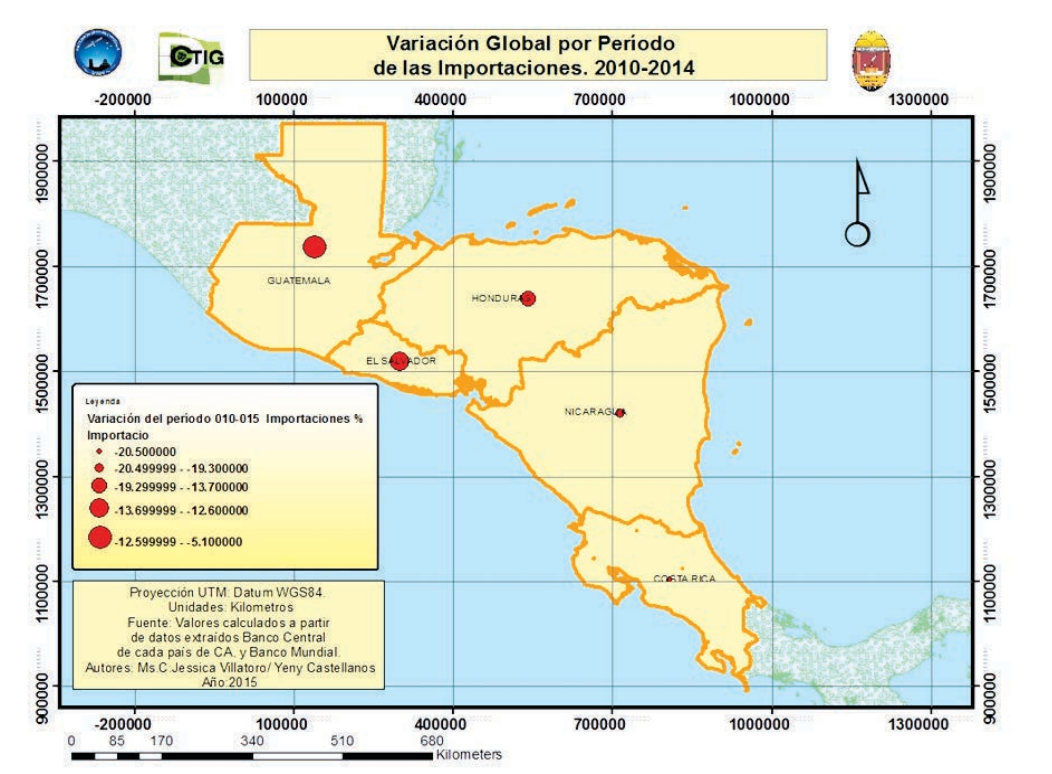

Figura 12. Se muestra que el consumo de bienes y servicios importados ha disminuido en toda la región Centroamericana sobre todo en Costa Rica (-20.5\%) y Nicaragua (-20.5, -19.3\%) 


\section{CONCLUSIONES}

- En términos generales se ha dado un rezago en la economía de la región Centroamericana debido a la crisis económica mundial que ha ocurrido durante el período 2010-2014, provocando una disminución en los niveles de producción y de consumo de la población e incrementando las tasas de desempleo de estos países. Aunado a esto un deterioro en la calidad de vida de la población y un estancamiento en el desarrollo humano.

- El País que más exportaciones tiene en comparación al resto de países estudiados en un período del 2010-2014 es Guatemala (-7.1\%, -1.4\%)

- El conjunto de variables: gasto de hogares, gasto de gobierno, inversión, exportaciones e importaciones permitieron medir la tendencia económica (PIB) de 5 países de Centroamérica, relacionados con la producción de un país, determinando que: Nicaragua obtuvo los mayores gastos y de forma general todos los países disminuyeron su inversión.

- Los niveles de producción, las exportaciones e importaciones y el consumo desempeñan un papel fundamental en la economía de un país. Los países que consumen mucho tienden a invertir poco, en cambio los que consumen poco tienden a invertir mucho.

- El consumo y la inversión pueden fluctuar de un año a otro, en el caso de inversión las variaciones pueden ser bastantes grandes. Lo anterior expresa que no está garantizado que el flujo total de gasto monetario, crecerá de una manera uniforme de un año a otro.

\section{BIBLIOGRAFÍA}

- Benito Muela, S. (s.f.). Teoria del Crecimiento Económico. En Apuntes de Macroeconomía IV. Madrid: UNED.

- Consejo monetrio centroamericano, Secretaia Ejecutiva. (s.f.). Determinantes del crecimiento economico en Centroamérica y Republica Dominicana. San Jose.

- Doménech, R. (2004). Política Fiscal y Crecimiento económico. Valencia, España. 
- Klein, L. (s.f.). Análisis económico de datos geográficos: técnicas de econometría espacial y geomarketing. Madrid.

- Klein, L. (S/F). Análisis económico de datos geográficos: técnicas de econometría espacial y geomarketing. Madrid, España.

- $\quad$ Madruga Torres, A., Tores Perez, M., Carballosa Torres, R., \& Perez Romero, A. (2007). Las asignaturas macro y microeconomía a la luz de la teoría Marxista, Leninista.

- Martín Moreno, J. (2006). Universidad de Vigo. Obtenido de http://webs. uvigo.es/jmartin/macroeconomiall/index_archivos/Tema4\%20ciclos $\% 20$ econ\%C3\%B3micos.pdf

- Mena, C. (2008). Exactitud espacial en la creacion de bases de datos SIG modelos raster y vectorial. Revista Chilena e Ingeniería, 159-168.

- Parkin, M., Esquivel, G., \& Muñoz, M. (2008). Economía, versión para Latinoamérica. México: Pearson.

- PEN. (2013). Estadisticas de Centro América, Indicadoes sobre desarollo humano sostenible. San Jose, Costa Rica.

- Sicori. (2011). Sistema corporativo de informacion geografica de PEMEX. Los SIG en la administracion publica. 